\title{
BMJ Open Effect of dose administration aids on adherence to self-administered medications: a systematic review protocol
}

\author{
Kanika Chaudhri (D) , ${ }^{1,2,3}$ Madeleine Kearney, ${ }^{1}$ Richard O Day (D) , 2,3 \\ Anthony Rodgers, ${ }^{1,2}$ Emily Atkins ${ }^{1,2,4}$
}

To cite: Chaudhri K, Kearney M, Day R0, et al. Effect of dose administration aids on adherence to self-administered medications: a systematic review protocol. BMJ Open 2019;9:e030536. doi:10.1136/ bmjopen-2019-030536

- Prepublication history and additional material for this paper are available online. To view these files, please visit the journal online (http://dx.doi. org/10.1136/bmjopen-2019030536).

Received 19 March 2019 Revised 23 July 2019

Accepted 20 August 2019

A Check for updates

C Author(s) (or their employer(s)) 2019. Re-use permitted under CC BY-NC. No commercial re-use. See rights and permissions. Published by BMJ.

${ }^{1}$ Cardiovascular Division, The George Institute for Global Health, Sydney, New South

Wales, Australia

${ }^{2}$ Faculty of Medicine, University of New South Wales, Sydney, New South Wales, Australia ${ }^{3}$ Department of Clinical Pharmacology and Toxicology, St Vincents Hospital Sydney, Darlinghurst, New South Wales, Australia

${ }^{4}$ Westmead Clinical School, University of Sydney, Sydney, New South Wales, Australia

Correspondence to Kanika Chaudhri; kchaudhri@georgeinstitute. org.au

\section{ABSTRACT}

Introduction Forgetting to take a medication is the most common reason for non-adherence to self-administered medication. Dose administration aids (DAAs) are a simple and common solution to improve unintentional non-adherence for oral tablets. DAAs can be in the form of compartmentalised pill boxes, automated medication dispensing devices, blister packs and sachets packets. This protocol aims to outline the methods that will be used in a systematic review of the current literature to assess the impact of DAAs on adherence to medications and health outcomes.

Methods and analysis Randomised controlled trials will be identified through electronic searches in databases including EMBASE, MEDLINE, CINAHL and the Cochrane Library, from the beginning of each database until January 2020. Two reviewers will independently screen studies and extract data using the standardised forms. Data extracted will include general study information, characteristics of the study, participant characteristics, intervention characteristics and outcomes. Primary outcome is to assess the effects of DAAs on medication adherence. Secondary outcome is to evaluate the changes in health outcomes. The risk of bias will be ascertained by two reviewers in parallel using The Cochrane Risk of Bias Tool. A meta-analysis will be performed if data are homogenous. Ethics and dissemination Ethics approval will not be required for this study. The results of the review described within this protocol will be disseminated through publication in a peer-reviewed journal and relevant conference presentations.

PROSPERO registration number CRD42018096087

\section{BACKGROUND}

Adherence is the extent to which a person's behaviour, specifically towards taking medication, corresponds with agreed recommendations from a healthcare provider. ${ }^{1}$ The effect of a medication and its long-term benefits depends on patient's adherence to prescriber instructions. Several factors contribute to the extent of medication adherence, however the most common reason provided for non-adherence is unintentional forgetfulness. ${ }^{2}$

\section{Strengths and limitations of this study}

- A comprehensive search strategy has been developed, in order to identify the majority of relevant and available literature.

- A thorough data extraction table will be used to maximise information gained from relevant studies.

- Sensitivity may be sacrificed to some extent in favour of a more precise search that will retrieve a manageable number of references.

Unintentional non-adherence is when an individual is unconsciously non-compliant with the directions they have received.

Generally, $40 \%$ to $60 \%$ of patients fail to consume medications as prescribed. ${ }^{3-5}$ Taking the right dose at the right time is commonly required for efficacy and safety. The duration of medication consumption can vary from a few days to several months or years. Medication adherence problems may be seen in both time frames, however the longer the duration of prescription, the more time there is for non-adherence to manifest. ${ }^{6}$ It has been observed that people taking medications long-term are more likely to miss doses particularly when the medication regimen is complex, or the disease being treated is asymptomatic. ${ }^{7}$

Unsurprisingly, adherence to a prescribed medication is lower when the drug is self-administered. ${ }^{8}$ Medication adherence is further threatened with the use of multiple medications, concurrent use of five or more medications is considered 'polypharmacy'. ${ }^{10}$ Polypharmacy is common in elderly people, making them a vulnerable group for medication non-adherence. Interventions to reduce the complexity of self-administration are needed and dose administration aids (DAAs) can be used to improve adherence to prescriptions. DAAs include compartmentalised plastic boxes, automated medication dispensing devices, blister packs and sachets 
packets. These aids assists patients in managing their medicines by arranging individual doses according to the prescribed time of administration.

DAAs can be assembled in a number of ways. Compartmentalised plastic boxes and automated medication dispensing devices are generally re-usable and can be pre-filled by a pharmacist, other healthcare worker, carer or the patient themselves. Blister or sachet packs are pre-packed and not re-usable. Repackaging of a medicine requires removal from its primary packaging, thus invalidates the stability guarantee by the manufacturer. However, it has been reported that drugs repackaged within DAAs have remained physically and chemically stable. ${ }^{11}$ Past literature has primarily focused on improving medication adherence to disease specific medications, rather than medications adherence overall. ${ }^{12-14}$ There have been examinations of a specific intervention such as the effect of a particular pillbox rather than all DAAs. ${ }^{15}$ Some reviews have focused on a specific population such as the elderly. ${ }^{16}{ }^{17}$ Other reviews have looked at interventions to improve medication adherence using DAAs but focused on the roles of pharmacists ${ }^{18}$ nurses $^{19}$ and physicians $^{20}$ in their use. A Cochrane review published in 2011 explored various different types of reminder packaging, including DAAs, for a broad range of formulations, but has not been updated since. ${ }^{6}$ In the absence of a current systematic review that focuses on adherence related to DAAs, healthcare professionals might conduct their own ad hoc reviews which likely lack sensitivity and may introduce bias. Our aim is to summarise the literature measuring the effect of medication adherence associated with DAAs.

\section{METHODS AND ANALYSIS}

This systematic review will include randomised control trials (RCTs) which incorporate DAAs and measure patient's adherence to medications. We have described our methods as per the Preferred Reporting Items for Systematic Review and Meta-Analysis for protocol (PRISMA-P) recommendations (online supplementary file 1). Final reporting of this study will be compliant with the main PRISMA statement.

\section{Patients and public involvement}

Patients and public were not involved in writing this protocol.

\section{Eligibility criteria}

Study design

In conducting the review discussed within this protocol, RCTs published in English will be considered eligible. Articles will be excluded if they are not a journal article, not based on empirical research (eg, protocol, editorial) and not reported in English. This review will include studies from the beginning of databases until January 2020.
It should be noted that the studies must contain sufficient detail, such as the criteria outlined within our data extraction form (online supplementary file 2), to be included within the review. For this reason, conference and poster abstracts, without full text papers, will be excluded from analysis. However, study authors will search databases for publications relating to the abstract.

\section{Types of participants}

There will be no restriction in participant characteristics. Participant can include any person, over the age of 18 , using a DAA.

\section{Types of interventions and comparators}

Intervention includes use of a DAA to assist with medication adherence. DAAs can include compartmentalised plastic boxes, automated medication dispensing devices, blister packs and sachet packets designed to organise pills according to when they should be taken. We excluded electronic (such as text messages) or mailed reminders to take medicines. However, use of DAAs can be supplemented by electronic or mailed reminders. Injected, topical or inhaled medications were excluded. Comparator will be usual care.

\section{Study outcomes}

The primary outcome of this study is to assess the effect of DAAs on patient's adherence to medications, measured through pill count or self-reporting. The secondary outcome is to assess the changes in health outcomes (such as change in blood pressure for hypertension) as a result of implementing use of DAAs. The study must assess the primary outcome to be deemed eligible for the review.

\section{Search strategy}

This systematic review will involve searches of MEDLINE, EMBASE, CINAHL and Cochrane databases. Further studies will be obtained from scanning reference lists of relevant studies, including other systematic reviews, and citation searching of key papers identified for inclusion. Any study published from the inception of the databases, prior to January 2020 will be included in the review. The search will be updated prior to submission of the review. A search strategy was developed with the initial support of a medical research librarian (online supplementary file 3).

\section{Data management}

After searching, the articles, including their abstracts as well as the full texts, will be exported to Endnote V.X9 (Thomson Reuters, New York, USA) for storage. Articles will be stored on a password-protected server-based platform that is accessible to both reviewers. At each stage of the article selection process (eg, after consolidation of all articles prior to assessing eligibility based on title and abstract), back-up files of the Endnote database will be made in order to retrace any steps as needed in the review process. 


\section{Selection process}

Two researchers will undertake study selection independently. In the initial screening stage, these authors will conduct a title and abstract search to identify papers that potentially meet the criteria for study selection. For papers where it is unclear whether the study should be included, a further assessment against the criteria will be undertaken using the full text of these articles. Discrepant opinions between the two reviewers will be resolved in discussion with the senior author. The flow of studies through selection process, together with reasons for exclusion at the full-text review stage will be reported using a modified PRISMA diagram.

Due to time and resource constraints, for the purpose of this study authors will not be contacted to obtain further details when there is missing formation in the study report. These studies will be excluded but documented as a 'potentially applicable study'. Studies will not be excluded on funding source if inclusion criteria are met.

\section{Data collection process}

Once the studies for inclusion have been identified, information outlined in the standardised data extraction form (online supplementary file 2) will be collected from all included studies. The form will be piloted and optimised by the two reviewers using three randomly selected studies that satisfy the eligibility criteria. One author will independently extract data from the remainder of the included studies. The data extracted will be checked and verified by a second author.

\section{Data items}

The following data will be extracted from the included studies:

1. General study information: study title \& citation, study authors, year of publication and publication journal.

2. Characteristics of the study: aim or objectives of the study and study design.

3. Participant characteristics: numbers of participants and baseline demographics.

4. Intervention characteristics: type of dose aid, duration of the intervention and any coexisting interventions implemented.

5. Outcomes: result of primary outcome and statistical significance, statistical methods used, documentation of specific quantitative and qualitative outcomes of interest, risk of bias, adverse effects and study conclusion.

\section{Risk of bias in individual studies}

The risk of bias will be ascertained by two reviewers in parallel using The Cochrane Risk of Bias Tool. ${ }^{21}$ The assessment will be performed at study level and will focus on selection, performance, detection, attrition and reporting bias. Discrepant opinions between the two reviewers, regarding risk of bias, will be resolved in discussion with the senior author. The risk of bias for each study included will be taken into consideration during data synthesis. Sensitivity analysis will determine the impact of studies with high risk of bias on result. Additionally, Grades of Recommendation, Assessment, Development and Evaluation (GRADE) system will be used to summarise the quality of evidence for each outcome. ${ }^{22}$

\section{Data synthesis and analysis}

Data will be presented in a descriptive narrative and supplemented with tables and figures where appropriate. All papers regardless of quality determined by risk of bias and GRADE system will be included within the narrative synthesis. Meta-analysis will be performed if variables assessed in the selected papers are comparable and there is sufficient data. This will exclude the low quality papers.

\section{ETHICS AND DISSEMINATION}

This study does not require ethics approval. The results of the systematic review described within this protocol will be disseminated through publication in peer-reviewed journals and presentation at relevant conferences.

Acknowledgements Dr Wendy Lau for assisting with the initial screening of texts.

Contributors KC conducted the scoping searches, designed and piloted the forms, drafted the manuscript and manages the overall study. KC will be involved in study selection, data extraction, synthesis and analysis. MK will be involved in study selection, data extraction, synthesis and analysis. EA, ROD and AR conceived the study. All authors read and approved the manuscript.

Funding This systematic review forms part of KC's thesis and is not externally funded or commissioned.

Competing interests None declared.

Patient consent for publication Not required.

Provenance and peer review Not commissioned; externally peer reviewed.

Open access This is an open access article distributed in accordance with the Creative Commons Attribution Non Commercial (CC BY-NC 4.0) license, which permits others to distribute, remix, adapt, build upon this work non-commercially, and license their derivative works on different terms, provided the original work is properly cited, appropriate credit is given, any changes made indicated, and the use is non-commercial. See: http://creativecommons.org/licenses/by-nc/4.0/.

ORCID iDs

Kanika Chaudhri https://orcid.org/0000-0002-3453-0769

Richard 0 Day http://orcid.org/0000-0002-6045-6937

\section{REFERENCES}

1. Sabaté E. Adherence to long-term therapies: evidence for action. World Health Organization, 2003.

2. Chesney MA, Ickovics JR, Chambers DB, et al. Self-reported adherence to antiretroviral medications among participants in HIV clinical trials: the AACTG adherence instruments. AIDS Care 2000;12:255-66.

3. Stewart RB, Cluff LE. A review of medication errors and compliance in ambulant patients. Clin Pharmacol Ther 1972;13:463-8.

4. Degli Esposti L, Degli Esposti E, Valpiani G, et al. A retrospective, population-based analysis of persistence with antihypertensive drug therapy in primary care practice in Italy. Clin Ther 2002;24:1347-57.

5. Jones JK, Gorkin L, Lian JF, et al. Discontinuation of and changes in treatment after start of new courses of antihypertensive drugs: a study of a United Kingdom population. BMJ 1995;311:293-5.

6. Mahtani KR, Heneghan CJ, Glasziou PP, et al. Reminder packaging for improving adherence to self-administered long-term medications. Cochrane Database of Systematic Reviews 2011;36.

7. Martin LR, Williams SL, Haskard KB, et al. The challenge of patient adherence. Ther Clin Risk Manag 2005;1:189. 
8. Dixon WM, Stradling P, Wootton IDP. Outpatient PAS therapy. The Lancet 1957;270:871-2.

9. Cross AJ, Elliott RA, George J, et al. Interventions for improving medication-taking ability and adherence in older adults prescribed multiple medications. Cochrane Database Syst Rev 2016;20.

10. Linjakumpu T, Hartikainen S, Klaukka T, et al. Use of medications and polypharmacy are increasing among the elderly. J Clin Epidemiol 2002;55:809-17.

11. Tan JZY, Kwan YH, YHJSpj K. Stability of chronic medicines in dosage administration AIDS. How much have been done? Saudi Pharm J 2016;24:21-8.

12. Gomes M, Wayling S, Pang L. Interventions to improve the use of antimalarials in south-east Asia: an overview. Bull World Health Organ 1998;76:9-19.

13. Orton LC, Barnish G. Unit-dose packaged drugs for treating malaria. Cochrane Database of Systematic Reviews 2009;4:CD004614.

14. Wiseman LA, Calendar WLA. Calendar (blister) packs for multiple drug therapy in leprosy: an inexpensive, locally-produced version. Lepr Rev 1987;58:85-7.

15. Bartlett Ellis RJ, Knisely MR, Boyer K, et al. Pillbox intervention fidelity in medication adherence research: a systematic review. Nurs Outlook 2017;65:464-76.
16. Edirisinghe S, Raimi-Abraham BT, Gilmartin JF-M, et al. MultiCompartment compliance aids (MCAs): application to the geriatric community. Eur Geriatr Med 2015;6:65-8.

17. Cramer JA. Enhancing patient compliance in the elderly. Role of packaging AIDS and monitoring. Drugs Aging 1998;12:7-15.

18. Lindenmeyer $\mathrm{A}$, Hearnshaw $\mathrm{H}$, Vermeire $\mathrm{E}$, et al. Interventions to improve adherence to medication in people with type 2 diabetes mellitus: a review of the literature on the role of pharmacists. $J$ Clin Pharm Ther 2006;31:409-19.

19. Burhenn P, Smudde J. Using tools and technology to promote education and adherence to oral agents for cancer. Clin J Oncol Nurs 2015;19:53-9.

20. Peck CL, King NJ. Increasing patient compliance with prescriptions. JAMA 1982;248:2874-8.

21. Higgins JPT, Altman DG, Gøtzsche PC, et al. The Cochrane collaboration's tool for assessing risk of bias in randomised trials. BMJ 2011;343:d5928.

22. Guyatt G, Oxman AD, Akl EA, et al. Grade guidelines: 1. IntroductionGRADE evidence profiles and summary of findings tables. J Clin Epidemiol 2011;64:383-94. 